\title{
Preface of the Special Issue International Symposium “Worlds of Entanglement"
}

\author{
Diederik Aerts $^{1} \cdot$ Massimiliano Sassoli de Bianchi ${ }^{1,2} \cdot$ Sandro Sozzo $^{3} \cdot$ Tomas Veloz $^{1,4}$
}

Published online: 4 November 2019

(c) Springer Nature B.V. 2019

This special issue is one of the outcomes of the International Symposium "Worlds of Entanglement," held at the Free University of Brussels (VUB), on September 29-30, 2017. The event gathered more than 50 scholars from different disciplines, ranging from pure mathematics to visual arts, and from multiple parts of the world, including Argentina, Austria, Canada, Chile, France, Germany, Italy, Japan, Poland and the United States, among others, to animate an interdisciplinary dialogue about fundamental issues of science and society.

Entanglement is a quantum phenomenon that can be produced when two or more physical entities interact, and is reflected in the impossibility of describing the obtained collection in terms only of the composing entities (Horodecki et al. 2009). Indeed, the only possible description is in terms of a "global state" which encodes the properties of all the entangled entities as a whole, as if forming a bigger interconnected single entity. Quantum entanglement has no counterpart in classical physics, which led one of the founding fathers of quantum theory, Erwin Schrödinger, to consider it as not "one but rather the characteristic trait of quantum mechanics, the one that enforces its entire departure from classical lines of thought" (Schrödinger 1935).

One of the fundamental peculiarities of quantum entanglement is that, when one of the entities undergoes a measurement, and its state changes according to the obtained outcome, all the other entities which were previously entangled with it will also collapse to states that are correlated to each other. And even when the entities are separated by large spatial

Tomas Veloz

tveloz@gmail.com

Diederik Aerts

diraerts@vub.ac.be

Massimiliano Sassoli de Bianchi msassoli@vub.ac.be

Sandro Sozzo

s831@leicester.ac.uk

1 CLEA, Brussels Free University, Brussels, Belgium

2 Laboratorio di Autoricerca di Base, Lugano, Switzerland

3 School of Business and Centre IQSCS, University of Leicester, Leicester, United Kingdom

4 Fundación para el Desarrollo Interdisciplinario de la Ciencia, la Tecnología y las Artes, Santiago, Chile 
distances, this effect of joint collapse appears to be instantaneous, hence cannot be the consequence of some information transmitted across space at finite speed. This seems to contradict one of the fundamental principles of relativity, which states that no signal can propagate in space faster than the limit speed of light, but many mechanisms are also in place to protect quantum theory from an actual breach of causality and in fact the phenomenon of entanglement, like also other quantum phenomena, suggests that not all we measure in our physics laboratories would be the result of processes unfloding exclusively within our spatiotemporal theater. In other words, entanglement, one of the fundamental features of the microscopic realm, nowadays massively applied in the development of new technologies, is challenging our most profound intuitions about the nature of our physical reality.

The general notion of entanglement was taken as a central idea for this meeting in Brussels mainly for two reasons. The first is that entanglement entails a notion of wholeness, which on the one hand calls into question the assumption that knowledge and information would be always decomposable into parts, and on the other hand explains that whatever decomposition into parts we choose, it can be expected that the obtained parts will be connected in some way and therefore still influence each other. This invites us, among other things, to rethink the way knowledge is created by the joint work of scholars. They tend to work and develop their careers enclosed in their disciplines or interacting very little with other disciplines in order to reach their specific research goals. Hence, scholars usually behave like non-entangled (classical) entities. However, by disconnecting from the advances and understandings of other disciplines, they can miss potentially important cross-fertilizations, and more important, they can miss larger encompassing questions such as how their research can serve the very advance of science or of society as a whole. In that sense, entanglement can also be taken as a powerful metaphor to propose novel ways of knowledge integration, by means of an interdisciplinary collaboration and the consequent creation of solid bridges between the different disciplines.

The second reason is not metaphorical but historical. We are now facing a second quantum revolution which opens up the application of quantum theory in radically novel ways with respect to the previous century (Dowling and Milburn 2003). In the first quantum revolution era, technologies such as laser and MRI were built upon fundamental quantum mechanical features such as indistinguishability and state excitation and a passive use of them was essentially made, in the sense that quantum features were mostly exploited in a "reading modality." The second quantum revolution, instead, also actively created and artfully manipulated quantum states and quantum contexts to produce useful and specific new applications. Among them, we find quantum cryptography, quantum computation and quantum metrology. Hence, the informational and structural possibilities of quantum systems have now become the core of todays' quantum technologies. In addition, a vast amount of research is devoted to identify new areas in which quantum-like theoretical structures can be found (Khrennikov 2014). These fields do not build quantum-like theories based solely on the fact that nature would be fundamentally constituted of quantum entities at the micro-level, but propose that quantum mechanisms are also in force in areas of scientific inquiry extending beyond the microscopic realm. In particular, the mathematical formalism of quantum theory, involving particular forms for state representation, probability calculus and time-evolution, can be extended to domains such as information retrieval, biology, cognition, social science and even evolutionary theories. Therefore, entanglement, as one of the core concepts of quantum theory, also possibly becomes a central feature of novel scientific approaches to traditional fields of scientific investigation.

But coming to the content of this special issue, its articles cover an interesting spectrum of topics relating various disciplines to quantum theory and in particular to the notion 
of entanglement. The first two articles are about foundational aspects of quantum theory (Diederik Aerts et. al, offer a review of the conceptuality interpretation of quantum theory, and Christian de Ronde, discusses the controversies related to quantum contextuality). Three articles propose novel languages to represent quantum structures (Claudio Garola, deals with contextual probabilities, Giuseppe Sergioli, with computational logic, and Massimiliano Sassoli de Bianchi, presents an accessible version of the extended Bloch representation of quantum mechanics). Three articles explore cognition and counsciousness (Chetan Prakash, on the interface theory of perception, Yukio Pegio-Gunji, on the relation between free-will and entanglement, and Jakub Tešar, on modeling game theoretical scenarios using quantum structures). Two articles also examine aspects relating to the foundations of physics (Jan Naudts, proposes the emergence of Coulomb forces from Quantum Electrodynamics, and Karl Svozil, discusses the possibility of unified physical theories by means of a high-dimensional fractal space-time). One article is about the interplay between Art and Science (Kyoko Nakamura, discusses the relation between entanglement and creativity). One article is about philosophy of language (Federica Russo) and another one is about ecological modeling and quantum structures (Tomas Veloz). ${ }^{1}$

Worlds of Entanglement was the natural continuation of past events organized by the Center Leo Apostel for Interdisciplinary Studies, around interdisciplinary reflection, in particular the "Einstein meets Magritte" Conference (1995), and the "Times of Entanglement" Symposium (2010), which brought together some of the world's most renowned thinkers, such as Zygmunt Bauman, Brian Arthur, Ilya Prigogine, Heinz von Foerster, William Calvin, Bas van Fraassen, Bob Edwards, Adolf Grunbaum, Rom Harré, Chris Langton, Constantin Piron, Francisco Varela, Linda Schele, Robert Pirsig, Barbara HernsteinSmith, John Ziman.

To conclude, we would like to thank some of the people who helped tremendously in the organization of the event: Ema Garboni, Karin Verelst, Clement Vidal, Cadell Last, Michel Barros Bessone, and Liesbeth Fieremans.

We hope to make this beautiful "Worlds of Entanglement" initiative further grow, organizing new interesting interdisciplinary meetings in a nextcoming future.

\section{References}

Dowling, J. P., \& Milburn, G. J. (2003). Quantum technology: The second quantum revolution. Philosophical Transactions of the Royal Society of London. Series A: Mathematical, Physical and Engineering Sciences, 361(1809), 1655-1674.

Horodecki, R., Horodecki, P., Horodecki, M., \& Horodecki, K. (2009). Quantum entanglement. Reviews of Modern Physics, 81(2), 865.

https://www.youtube.com/channel/UCKaagmCRZ84qF7xiBF93yQw

Khrennikov, A. Y. (2014). Ubiquitous quantum structure. Berlin: Springer.

Schrödinger, E. (1935). Discussion of probability relations between separated systems. Mathematical Proceedings of the Cambridge Philosophical Society, 31, 555-563.

Publisher's Note Springer Nature remains neutral with regard to jurisdictional claims in published maps and institutional affiliations.

\footnotetext{
${ }^{1}$ Lectures given at Worlds of Entanglement associated to these articles can be found in the Worlds of Entanglement YouTube channel (https://www.youtube.com/channel/UCKaagmCRZ84qF7xiBF93yQw).
} 
Diederik Aerts is professor at the Vrije Universiteit Brussel (VUB) and founding director of the Leo Apostel Centre for Interdisciplinary Studies (CLEA). He is Editor-in-Chief of the international ISI and Springer journal Foundations of Science (FOS) and a board member of the Worldviews group, founded by the philosopher Leo Apostel. He is president of the Centre for Quantum Social and Cognitive Science (IQSCS) at Leices- ter University (UK), and is a Fellow of the College of the International Institute for Advanced Studies in Systems Research and Cybernetics (IIAS). He was the scientific and artistic coordinator of the Einstein meets Magritte conference in 1995, where the world's leading scientists and artists gathered to reflect about science, nature, human action and society. CLEA was invited and participated in the World-Exhibition in Shanghai in 2010, with a follow up of this conference entitled 'Times of Entanglement'. Diederik Aerts started his research with a focus on the foundations of quantum physics and, during the last two decades, also on cognitive science. In this respect, he is considered a pioneer of the research domain called quantum cognition.

Massimiliano Sassoli de Bianchi graduated in physics from the University of Lausanne (UNIL), Switzerland, in 1989. From 1990 to 1991, he was an Assistant in the Department of Theoretical Physics (DPT) of the University of Geneva (UNIGE). In 1992, he joined the Institute of Theoretical Physics (IPT) at the Federal Institute of Technology in Lausanne (EPFL), as a Ph.D. candidate. He received the Ph.D. degree in physics from EPFL in 1995. Since 1996, he has been working in the private sector and as an independent researcher. In 2016, he joined the Center Leo Apostel for Interdisciplinary Studies (CLEA), at the Vrije Universiteit Brussel (VUB), as a research fellow. He is also the director of the Laboratorio di Autoricerca di Base (LAB), in Lugano, Switzerland, and the Editor of the Italian journal AutoRicerca. His research activities are focused on the foundations of physics, quantum cognition and consciousness studies. He has written essays, popular science books, children's stories, and published numerous research articles in international journals.

Sandro Sozzo is associate professor at the University of Leicester. His research interests mainly concern the development of novel mathematical models of judgment and decision-making under uncertainty, with relevant applications in behavioral economics and finance. He is author of more than 80 publications, organ- ized conferences, managed special issues as guest editor, and was invited to give lectures in UK (Oxford), US, Canada, EU and China. He is the director of the "Centre for Quantum Social and Cognitive Science" (IQSCS), the secretary of the "International Quantum Structures Association" and the managing edi- tor of the Springer Nature journal "Foundations of Science". Currently, he is partner of the consortium "QUARTZ", involving seven European universities, which has recently been funded for a Marie Curie Innovative Training Network.

Tomas Veloz graduated in physics (B.Sc. 2004), in mathematics (B.Sc. 2008), and computer science (M.Sc. 2010), at the University of Chile. He obtained a Ph.D. in interdisciplinary studies at the University of British Columbia, Canada (2015), did 1-year postdoc at the Centre Leo Apostel for Interdisciplinary Studies (CLEA), at the Vrije Universiteit Brussel (VUB), where still maintains affiliation as research fellow, and is currently doing a 3-year postdoc at the Institute of Philosophy and Complexity Sciences (IFICC - Chile), where he also works as director of the systemics department. His research focuses in mathematical modeling of interdisciplinary processes, with emphasis in quantum models applied to cognitive situations and reaction network models for system-theoretic models in biology and social systems. He is author of more than 25 articles, has organized international conferences, managed special issues as guest editor, and was invited to give lectures in various countries in EU and South America, as well as to UK, USA, Canada, and China. 